\title{
Instalación de fincas cafetaleras en sistema agroforestal para recuperación y sostenibilidad de suelos
} degradados de selva alta

\section{Installation of coffee farms in agroforestry system for recovery and sustainability of degraded soils of the high jungle}

\author{
Josué Otoniel Dilas-Jiménez ${ }^{* *}$, Carlos A. Mugruza-VassalloiD
}

\section{RESUMEN}

Los suelos ubicados en la zona ecológica de Región Yunga o Selva Alta, caracterizados como Bosque de terraza baja basimontano (Btb-ba) y Bosque de terraza alta basimontano (Bta-ba), con altitudes entre los 800 a $1200 \mathrm{msnm}$, generalmente poseen una cubierta forestal natural siendo particularmente vulnerables a la degradación del suelo, por lo que es necesario protegerlos. Un inadecuado manejo de estos suelos está generando su degradación, con la consecuente ampliación de la frontera agrícola y presión sobre nuevas áreas de bosque natural. Ante ello, se presenta una propuesta de finca cafetalera en sistema agroforestal (SAF), como alternativa ecológica y sostenible para suelos degradados adaptable a la provincia de Rioja. El SAF propuesto para ser aplicable en suelos degradados, se plantea en 2 procesos: i) Proceso de recuperación del suelo, por los dos primeros años con la instalación de un cobertura de suelo con Centrosema macrocarpum y la instalación de Inga edulis, así como de Tithonia diversifolia entre las ingas; ii) Proceso de implementación del SAF, por un periodo aproximado de 30 años con la instalación de árboles forestales como Colubrina glandulosa y de Cedrelinga catenaeformis a razón de $\sim 140$ individuos de cada especie por hectárea, así como la instalación de cafetal como generador de ingresos inmediatos. Este SAF genera como salidas, productos como: leña, madera rolliza, madera aserrada, café. Así también ayudo en la recuperación de la fertilidad del suelo y genera beneficios ambientales como la captura de carbono.

Palabras clave: sistemas agroforestales, café, selva alta, suelos degradados

\begin{abstract}
The soils located in the ecological zone of the yunga or high jungle, which is characterized as Basimontain low terrace forest (Btb-ba) and Basimontain high terrace forest (Bta-ba), With altitudes between 800 to 1200 masl, they are forests that have natural forest cover, being particularly vulnerable to soil degradation, which is why it is necessary to protect. An inadequate management of these soils has been generating their degradation with the consequent expansion of the agricultural frontier and pressure on new areas of natural forest. Given this, a proposal for a coffee farm in an agroforestry system (SAF) is presented as an ecological and sustainable alternative for degraded soils adaptable to the province of Rioja. The SAF proposed to be applicable in degraded soils, is installed in 2 processes: i) Soil recovery process, for the first two years with the installation of a ground cover with Centrosema macrocarpum and the installation of Inga edulis, as well as Tithonia diversifolia among the ingas; ii) SAF implementation process, for an approximate period of 30 years with the installation of forest trees such as Colubrina glandulosa and Cedrelinga catenaeformis with about $\sim 140$ individuals of each species per hectare, as well as the installation of coffee plantations as income generator immediate. This SAF generates outputs such as firewood, roundwood, sawnwood, coffee; Thus also the recovery of soil fertility and environmental benefits such as carbon capture.
\end{abstract}

Keywords: agroforestry system, coffee, high jungle, degraded soil, sustainability

\footnotetext{
${ }^{1}$ Universidad Nacional Autónoma de Tayacaja Daniel Hernández Morillo, Escuela Profesional de Ingeniería Forestal y Ambiental, Huancavelica, Perú

${ }^{2}$ Universidad Nacional Tecnológica de Lima Sur, Grupo de Investigación en Computación y Neurociencia Cognitiva, Lima, Perú

*Autor de Correspondencia, e-mailjdilas@unat.edu.pe
} 


\section{INTRODUCCIÓN}

La agricultura sustentable, se define como aquella que permite mantener en el tiempo un flujo de bienes y servicios que satisfagan las necesidades socioeconómicas y culturales de la población, dentro de los límites biofísicos que establece el correcto funcionamiento de los sistemas naturales (Sarandón et al., 2006). Si bien en países como Colombia se trabaja con cierta recuperación del suelo para el café (Suarez-Vasquez, 1994), Pretty (2007) indicó que el desafío actual de la sostenibilidad se centra en la necesidad de desarrollar tecnologías y prácticas agrícolas que i) no tenga efectos adversos en el ambiente, ii) accesible y efectivo para los agricultores, iii) contribuir a la seguridad alimentaria como tener efectos positivos en los bienes y servicios ambientales.

El café es el principal producto agrícola de exportación peruano, el cual tiene un gran impacto en la economía pues genera 43 millones de jornales al año, aunque su volumen de exportación no denota crecimiento sostenido, sino una reducción en el año 2019 y una mayor caída para el año 2020 (JNC, 2019). A nivel mundial el Perú es el séptimo país exportador y ocupa el segundo puesto en exportación de cafés orgánicos (MINAGRI, 2017). El café es producido por pequeños agricultores mediante agricultura tradicional porque usa recursos locales, sin embargo, por destinar su producción al mercado, hace uso de la agricultura moderna (Sánchez et al., 2017).

Como ejemplo de caso de suelos degradados de selva alta en Perú se tiene a la a región San Martín, específicamente las provincias de Rioja, Moyobamba y Lamas al ser las principales zonas cafetaleras (Dilas 2013). La zona cafetalera de Rioja, por un inadecuado manejo de los cafetales viene generando la degradación de suelos con la consecuente ampliación de la frontera agrícola y presión sobre nuevas áreas de bosque puntualmente en la zona de amortiguamiento, y el mismo Bosque de Protección Alto Mayo-BPAM, teniendo en cuenta que según estimaciones la deforestación en la región San Martín es aproximadamente de 30000 ha/año (Layza etal., 2018).
La zona cafetalera en la provincia de Rioja, se localiza puntualmente los distritos de Awajun y Pardo Miguel, y en la zona ecológica de la Región Yunga o Selva Alta, caracterizados como Bosque de terraza baja basimontano (Btb-ba) y Bosque de terraza alta basimontano (Bta-ba), con altitudes entre los 800 a $1200 \mathrm{msnm}$ (MINAM, 2015). Estudios realizados por Walentowski et al. (2018) en ecosistemas de selva alta colindantes al BPAM (Región Amazonas), indican que estos bosques poseen una cubierta forestal natural o casi natural siendo particularmente vulnerables a la degradación del suelo por lo que deben protegerse. Por tanto, es importante encontrar alternativas silvícolas que consideren la protección de los árboles y el manejo sostenible.

Según la FAO (2020), la degradación del suelo es el cambio en la salud del suelo resultando en una disminución de la capacidad del ecosistema para producir bienes o prestar servicios para sus beneficiarios. Es así, que, para la degradación de un suelo, el principal causante es el hombre a través del uso inadecuado del recurso, es por ello que la degradación, además de incluir la erosión por actividades humanas, también incluye la salinización, la solidificación, la acidificación, la intoxicación, la contaminación, el aumento en la compactación, la disminución de la infiltración, la conductividad hidráulica y la cantidad de humus existente (Kairis et al., 2014; Alegre et al., 2019). Incluso países con gran extensión geográfica y zonas mineras como el Brasil, que recientemente han publicado resultados de recuperación del suelo de café en muestras a los 2,5, 3,5, y 4,5 años de experimentación (Vilas-Boas et al., 2018).

Ante la problemática antes mencionada, se hace necesario generar propuestas que permitan hacer un adecuado uso de los recursos, puntualmente el uso del suelo para asegurar la sostenibilidad. Investigaciones realizadas en la provincia de Yurimaguas, región Loreto concluyen que los sistemas agroforestales (SAF) apropiados pueden mantener e inclusive reponer la fertilidad del nitrógeno a través de las bacterias fijadoras de nitrógeno, la captura de nutrientes en profundidad y varios mecanismos de reciclado (Alegre et al., 
2015). También se ha demostrado, que la agroforestería con árboles para sombra son una mejor alternativa para mantener la biodiversidad, que los monocultivos intensivos (Somarriba et al., 2012). Así, una de las ventajas atribuidas a la agroforestería es la mejora de la fertilidad del suelo a través de un reciclaje de nutrientes, recomendándose incluir siempre árboles o arbustos fijadores de nitrógeno (Sánchez 1987) como el Centrocema macrocarpum y la Tithonia diversifolia.

En tal sentido, el presente estudio busca generar una propuesta de finca cafetalera en sistema agroforestal como alternativa ecológica y sostenible para suelos de selva alta degradados por la agricultura como el cultivo de café, tomando como referencia a suelos degrada- dos de selva alta en la provincia de Rioja, Región San Martín, Perú.

\section{MATERIALES Y MÉTODOS}

\section{Búsqueda de información base}

Haciendo uso de motores de búsqueda en bases de información científica especializada como SCOPUS, SPRINGER y otras, se encontraron artículos científicos, a partir de los cuales se planteó una propuesta de sistema agroforestal adaptable para una zona de selva alta.

\section{Ubicación de la zona adaptable para la propuesta}

La presente propuesta de SAF se plantea para la zona de selva alta, con suelos degradados por la agricultura y la ganadería en la provincia de Rioja (Figura 1).

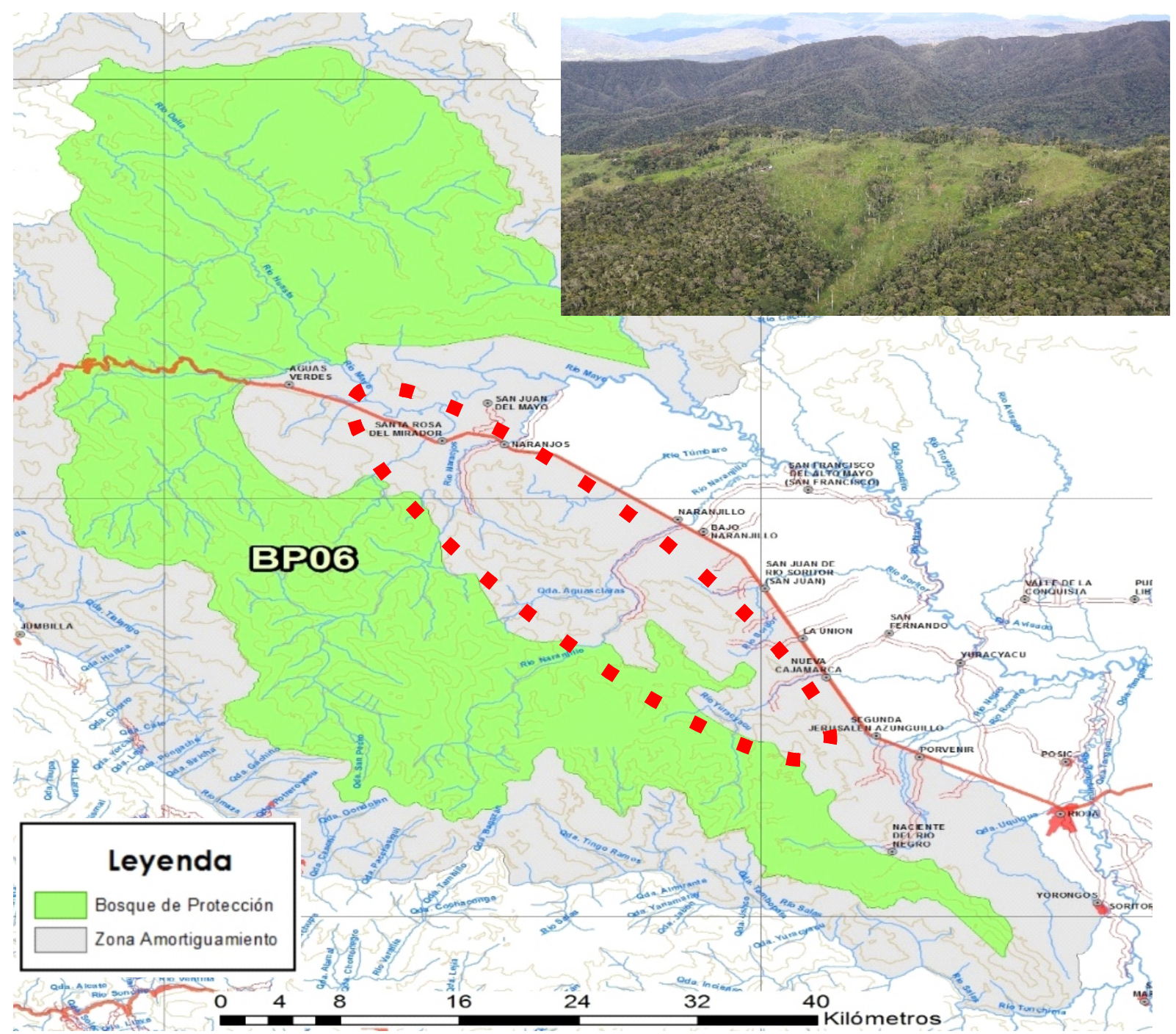

Figura 1. Zona de suelos degradados por el inadecuado manejo de la agricultura (línea punteada en rojo), provincia de Rioja. Fuente: SERNANP (2017). 


\section{Diseño e implementación del Sistema Agroforestal}

\section{Proceso de Recuperación del suelo (2 años)}

Para la recuperación del suelo degradado, luego de una limpieza del terreno, se plantea la instalación de Centrosema macrocarpum (centrosema), que durará todo el tiempo de vida del SAF, a razón de $2 \mathrm{~kg}$ de semilla botánica por hectárea, mediante siembra tradicional con azadón en distanciamiento de 50 x $50 \mathrm{~cm}$. Sin embargo, considerando el empobrecimiento de los suelos, se debe incorporar fósforo (P) al suelo, mediante la aplicación de roca fosfórica previo a la siembra del centrosema a razón de $20 \mathrm{~kg}$ P/ha (Arévalo et al., 2003). Está especie dará un importante aporte de nitrógeno (N) al suelo (Alegre, 2008), además de que puede constituir una importante fuente forrajera, para promover la diversificación productiva del pequeño agricultor (Hussain et al., 1988).

Considerando el rápido crecimiento del centrosema, y a fin de facilitar el reciclaje de nutrientes, a los 3 meses de haber instalado esta cobertura, se plantea la instalación de Inga edulis (guaba) con un distanciamiento de 3 x 6 m (Farfán et al., 2003). Asimismo, de manera complementaria para favorecer principalmente la fertilidad del suelo por la incorporación de fósforo intercambiable, potasio $(\mathrm{K})$ intercambiable, calcio (Ca) y magnesio $(\mathrm{Mg})$; favorecer la propiedades químicas del suelo con la incorporación de materia orgánica (Agbede et al., 2014; Theobald et al., 2014; Moriones y Montes 2017; Lotutala y Kimbembi, 2018) y dotar de protección al SAF, se plantea la instalación de plantas de Tithonia diversifolia (botón de oro), a razón de 2 plantas entre las guabas. Galindo et al. (2017) en un estudio realizado en los Andes Centrales de Colombia, encontraron que la $T$. diversifolia se comporta como arbusto nodriza (otorga protección) para las primeras etapas de crecimiento de los árboles plantados. Adicionalmente este arbusto puede constituir una importante fuente forrajera para promover la diversificación productiva del pequeño agricultor, ya que su contenido proteico supera el 20\% (Hohnwald et al., 2016).

Proceso de implementación del SAF
Considerando el desarrollo vegetativo de la guaba, la necesidad de aprovechar el reciclaje de nutrientes y la sombra para la instalación del cafetal y las especies forestales después de 2 años, se hará un primer raleo sistemático de las guabas. Se dejarán a un distanciamiento de 6 × 6 m en tresbolillo, en cuyos espacios se realizará la plantación de una especie forestal de rápido crecimiento como la Colubrina glandulosa (shaina) (Alegre, 2005; Craven et al., 2011; Gama et al., 2013; Marquardt et al., 2013). Además de que no necesita de condiciones especiales para su germinación y tiene un alto porcentaje de sobrevivencia (Silva et al., 2015; Pinto et al., 2020). También se plantea otra especie forestal valiosa y de mediano crecimiento como la Cedrelinga catenaeformis (tornillo) (Jarrett et al., 2017). El distanciamiento inicial de las especies forestales shaina y tornillo, sería de cada $12 \mathrm{~m}$ por fila en sistema intercalado ( $\sim 140$ plantas/ha), las filas de especies instaladas quedarían de manera intercalada (Figura 2).

En el surco que tiene un espacio de 6 metros, se instalará una fila doble de cafetal (Coffea arabica) con un distanciamiento de $1 \mathrm{~m}$ entre plantas y 1,5 m entre par de plantas (2220 plantas/ha) (Arcila, 2003). Entre las variedades de café recomendadas están el catimor, el castillo u otra tolerante a la roya del café y al cultivo bajo sombra (Julca et al., 2018; Milla et al., 2019).

Después de 2 años de la instalación del cafetal y las especies forestales (año 4 de la instalación del SAF), se hará un segundo raleo eliminando todas las guabas como aprovechamiento de leña, dando espacio al crecimiento de las especies forestales. Después de 6 años (10 años de la instalación del SAF) se hará un tercer raleo mediante el aprovechamiento de la shaina al $100 \%$, y a fin de promover la formación y desarrollo de los árboles de tornillo se realizará un raleo eliminando un $25 \%$ de estos árboles, quedando aproximadamente 100 árboles de tornillo por hectárea (Alvim y Nair 1986; Álvarez y Ríos, 2009).

Considerando que el cafetal es un cultivo de producción anual, y por tanto necesita un alto requerimiento nutricional. Se recomienda, que además del reciclaje 


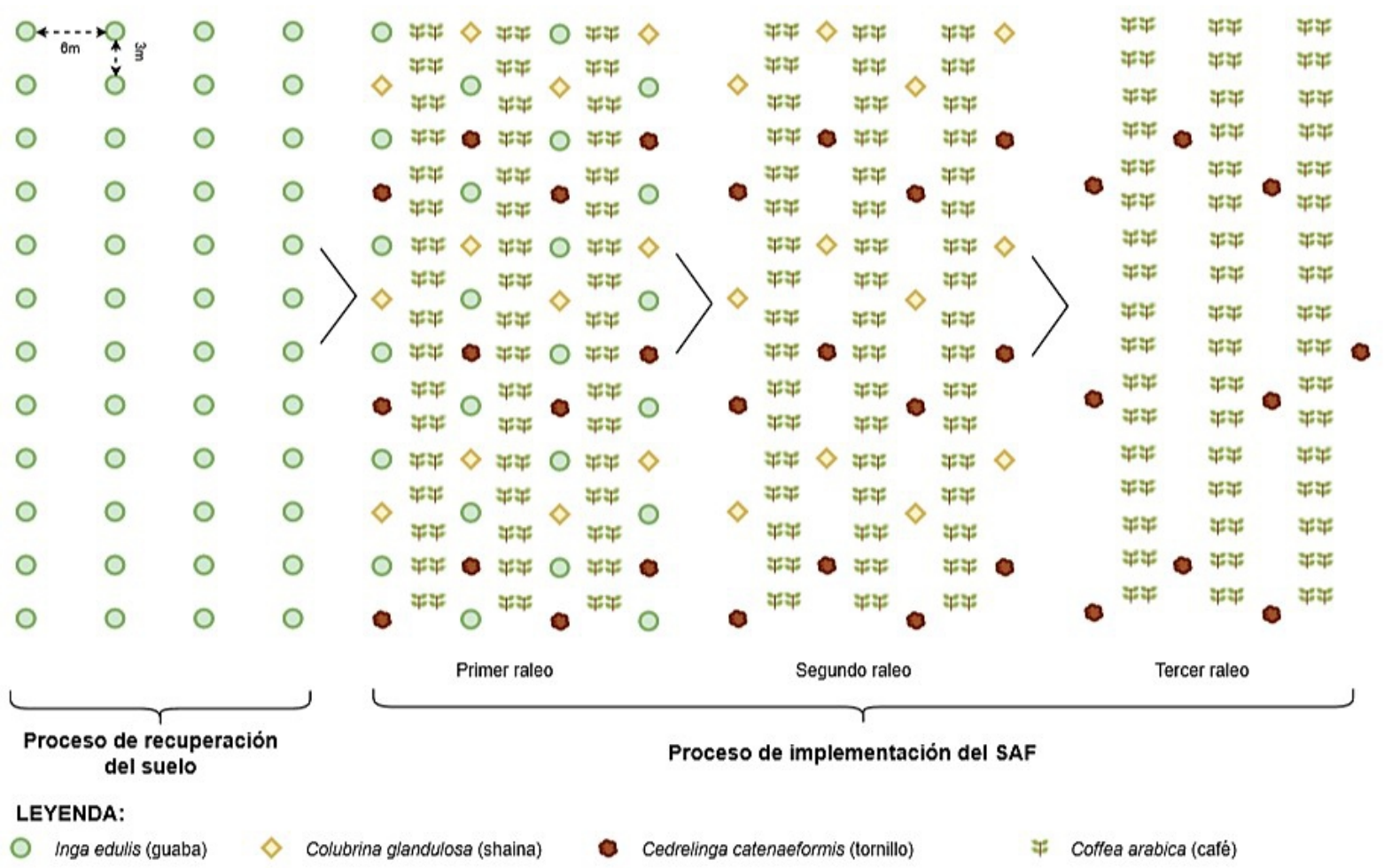

Figura 2. Diseño de implementación del SAF, mostrando los raleos propuestos.

de nutrientes aportados principalmente por la cobertura del centrosema y la $T$. diversifolia, y del aporte de los árboles en el SAF, se deberá complementar con abonos según el requerimiento nutricional del suelo. Principalmente, se deben usar abonos con $\mathrm{P}$ y N, así como enmiendas que favorezcan la mineralización del Py N (Neina et al., 2016). Por otro lado, considerando la curva de producción de cafetal, se recomienda que después de la séptima campaña (año 10 de la instalación del SAF) se implemente algún método de renovación del cafetal, por ejemplo, mediante poda sistemática para otro ciclo de producción.

\section{Evaluación de la sostenibilidad del SAF}

La evaluación de la sostenibilidad de fincas cafetaleras en SAF, se puede medir considerando varias variables en distintas dimensiones e indicadores para cada dimensión (Tabla 1). Para ello, se puede tomar como referencia lo utilizado por Sarandón (2002), Acuña (2008), Reyes (2017) y Alegre (2019).

\section{PROPUESTA DE INSTALACIÓN DELSAFY RESULTADOS ESPERADOS}

El arreglo del SAF propuesto en la metodología, que- daría con el diseño mostrado en la figura 2.

La productividad del cafetal instalado puede alcanzar entre 1000 a $1500 \mathrm{~kg} / \mathrm{ha}$, siendo menor a una producción de un cafetal instalado a pleno sol (alta demanda de insumos) (Arcila 2003). Sin embargo, por las características productivas, pueden ser factible obtener certificaciones de cafés sostenible o especiales y obtener precios superiores al café convencional. Además, se ha reportado que la cobertura arbórea favorece la calidad física y sensorial del café (Milla et al., 2019).

De otro lado, es preciso indicar que es necesario un adecuado manejo de la sombra en el cafetal. La sombra en el SAF podría favorecer la incidencia de plagas y enfermedades de la hoja como la Cercospora coffeicola (cercospora) y de Micena citricolor (minador del café) (Julca et al., 2010). Sin embargo, variedades de café como el castillo han mostrado un buen comportamiento agronómico en cultivo bajo sombra de especies forestales como la shaina (Milla et al., 2019)

Sobre la plantación de shaina, se plantea el aprovechamiento a los 8 años de edad, acorde a lo evaluado por Olivares y Torrejón (2007) en las provincias de Lamas 
Tabla 1. Variables e indicadores de sostenibilidad de finca cafetalera en sistema agroforestal

\begin{tabular}{|c|c|c|}
\hline Dimensión/ Indicador & Variable/Criterio & Unidad de medida \\
\hline \multicolumn{3}{|c|}{ Biofísica } \\
\hline \multirow{7}{*}{$\begin{array}{c}\text { Propiedades físicas, } \\
\text { químicas y biológicas } \\
\text { del suelo }\end{array}$} & Densidad aparente & $\mathrm{g} / \mathrm{cm}^{3}$ de $0-20 \mathrm{~cm}$ de profundidad \\
\hline & Porosidad & $\%$ porosidad \\
\hline & Materia orgánica & $\%$ de materia orgánica \\
\hline & Nitrógeno en el suelo & Ppm de nitrógeno \\
\hline & Fósforo en el suelo & Ppm de fósforo \\
\hline & & Macrofauna: Conteo de lombrices \\
\hline & Biología del suelo & $\begin{array}{c}\text { Microfauna: Conteo microbiano }(\mathrm{UFC} \times 100 \mathrm{~g} \text { de } \\
\text { suelo) }\end{array}$ \\
\hline \multicolumn{3}{|c|}{ Socioeconómica } \\
\hline Sociales & $\begin{array}{c}\text { Empleabilidad } \\
\text { Diversificación productiva }\end{array}$ & $\begin{array}{c}\mathrm{N}^{\circ} \text { jornales por ha por año } \\
\mathrm{N}^{\circ} \text { productos que se ofertan de la finca }\end{array}$ \\
\hline \multirow{3}{*}{ Económicos } & Producción de café & $\begin{array}{c}\text { Quintales/ha } \times \text { año (quintales de } 55,2 \mathrm{~kg} \text { de café } \\
\text { pergamino seco) }\end{array}$ \\
\hline & $\begin{array}{l}\text { Producción de madera } \\
\text { aserrada }\end{array}$ & $\begin{array}{c}\text { Pie tablar/ha } \times \text { año (pie tablar de madera aserrada, a } \\
\text { la cosecha). Tornillo }\end{array}$ \\
\hline & $\begin{array}{l}\text { Producción de madera } \\
\text { rolliza } \\
\end{array}$ & $\mathrm{N}^{\circ}$ troncos rollizos/ha (shaina y tornillo) \\
\hline \multicolumn{3}{|c|}{ Ambiental } \\
\hline Servicios ambientales & Captura de carbono & $\begin{array}{l}\text { Toneladas de } \mathrm{CO}_{2} \text { capturado/secuestrado por ha } \\
\text { (tanto en biomasa y suelo) }\end{array}$ \\
\hline
\end{tabular}

y San Martín. Se puede proyectar que con promedios de diámetro a altura de pecho (DAP) de $\sim 12 \mathrm{~cm}$ y una altura comercial de $\sim 9 \mathrm{~m}$, aplicando un cociente de forma de 0,7 (García et al., 2013) y la fórmula de Smalian $\left(\mathrm{V}=0,3927 \times\left(\mathrm{D} 1^{2}+\mathrm{d} 2^{2}\right) \times \mathrm{L}\right)$, una hectárea produciría $\sim 10 \mathrm{~m}^{3}$ de madera rolliza, o en su defecto considerando 3 troncos rollizos de $3 \mathrm{~m}$ de largo por árbol, se producirán cerca de 420 troncos. La venta de madera rolliza puede representar $\sim \mathrm{S} / 2300.00$ por hectárea al décimo año de la instalación del SAF. En la investigación realizada por Gama et al. (2013), la shaina logró una altura de 3,10 m en 24 meses, lo cual indica la alta velocidad de crecimiento y el potencial para ser cosechada en el periodo propuesto.

En cuanto a la producción de madera de tornillo, se puede considerar la cosecha a los 40 años, como la edad en la que alcanzaría el tamaño comercial (Rondon et al., 2009). Se puede proyectar que con promedios de DAP $\sim 70 \mathrm{~cm}$ y una altura comercial de $15 \mathrm{~m}$, aplicando un cociente de forma de 0,8 (Sánchez, 2012) y la fórmula de Smalian $\left(V=0,3927 \times\left(D 1^{2}+d 2^{2}\right) \times L\right)$ el SAF propuesto podría llegar a producir $\sim 650 \mathrm{~m}^{3}$ de madera rolliza que equivale a 183500 pies tablares, lo cual estaría dentro de los rangos de producción indica- do por Álvarez y Ríos (2009).

En cuanto a la captura de carbono (C) del SAF propuesto, este sistema podría capturar en la biomasa aérea y hojarasca más de 60 tC/ha y en el suelo alrededor de 40 tC/ha, acorde a Alegre (2017). Resultados de evaluación realizada por Núñez (2018), en la zona del Alto Mayo en la Región San Martín muestran que cada árbol de tornillo, con sólo $29 \mathrm{~cm}$ de DAP contiene $600 \mathrm{~kg}$ de biomasa aérea, representando $\sim 0,3$ tC. Para una densidad de 140 árboles de tornillo por hectárea, como se plantea en este SAF almacenarían al menos $40 \mathrm{tC} / \mathrm{ha}$ en la biomasa aérea. Esto sin considerar la biomasa aérea de la shaina así como el almacenamiento de $\mathrm{C}$ por parte del tornillo, que seguirá en aumento a medida que aumenta su tamaño. Por otra parte, hay estudios que han demostrado que la shaina aporta con importantes niveles de carbono orgánico en el suelo $(111 \mathrm{~g} / \mathrm{kg})$ que puede ser debida a su baja respiración basal (Weber et al., 2019). Para obtener la cantidad de dióxido de carbono $\left(\mathrm{CO}_{2}\right)$, necesario para el servicio intercambiable en los mercados de carbono se multiplicaría el valor del C almacenado por el factor de conversión 44/12 (De Morais, 2019).

Respecto a la recuperación del suelo degradado, 
teniendo en cuenta sus indicadores biofísicos, destaca el estudio realizado en el marco del proyecto VLIR.UOS de la Universidad Nacional Agraria La Molina (Alegre, 2008), en sistemas agroforestales multiestrato de tornillo y cobertura de centrosema instalados en Yurimaguas, Loreto. Este sistema aportó al suelo en promedio $150 \mathrm{~kg} \mathrm{~N} / \mathrm{ha} \times$ año. Asimismo, en un sistema silvopastoril en la misma zona a pesar del pastoreo se encontró un aporte de $40 \mathrm{~kg} \mathrm{P} / \mathrm{ha} \times$ año. Las plantas de guaba instaladas en el SAF aportaran hasta unos $40 \mathrm{~kg} \mathrm{~N} /$ ha×año (Sánchez, 1987), todo ello adicionado a que los insumos orgánicos tienen un efecto positivo en la diversidad microbiana (Kamaa, 2011)

Finalmente, al realizarse la implementación sistemática y adecuadamente documentada podemos agregar conocimiento de alto impacto, puesto que al realizar búsquedas en SCOPUS con las palabras clave en el Título o Resumen para ("Colubrina glandulosa" o "Cedrelinga catenaeformis") se encontraron 31 documentos, de los cuales solo 3 de ellos se referían a plantaciones de café, y ninguno de estos 3 artículo tenía un método de recuperación. De la misma forma buscamos en el Título y Resumen ("Colubrina glandulosa" o "Cedrelinga catenaeformis" o "Tithonia diversifolia") y (forest) encontrando 35 artículos y ninguno de ellos se refería al suelo del café.

\section{CONCLUSIONES}

El SAF propuesto busca su instalación y aplicación a zonas con suelos degradados en la selva peruana. Para ello se plantea un primer proceso de recuperación de suelo por un periodo mínimo de 2 años y luego la implementación misma del SAF. En total la propuesta puede tener un ciclo alrededor de los 30 años considerando la plantación de Cedrelinga catenaeformis (tornillo) en cuyo periodo se pueden hacer uno o más ciclos productivos de café, para cuyo cultivo se recomienda una renovación cada 7 campañas de producción.

El SAF planteado busca la recuperación de suelos degradados y la sostenibilidad del cultivo del café en la zona productora de Rioja, en la Regíon San Martín, el cual puede ser evaluado con indicadores en las dimensiones biofísicas, socioeconómicas y ambientales.

Por la salida de múltiples productos e incluso servicios ambientales que puede tener el SAF propuesta, se puede concluir que su implementación sería económica rentable, socialmente aplicable y ambientalmente amigable, y podría contribuir a frenar la actual presión al bosque natural.

A futuro, después de la implementación es posible que otras investigaciones manejen el compostaje de materiales orgánicos como de la pulpa del café, entre otros, para el mejoramiento del suelo. Por ejemplo, el proyecto del Fondo de Investigación y Desarrollo para la Competitividad -FIDECOM, sobre el manejo del compostaje de pulpa de café como abono orgánico (Dilas y Villanueva, 2014).

\section{V.AGRADECIMIENTOS}

El primer autor agradece a los ingenieros Giancarlo Orellana, Mónica Narrea y Jennefour Salazar quienes contribuyeron con importantes aportes a en la parte metodológica, puntualmente el diseño del SAF. Así también al Dr. Julio Alegre quién en el marco del Curso Planificación y Evaluación de Sistemas Sustentables del programa de Doctorado en Agricultura Sustentable de la Universidad Nacional Agraria La Molina, facilitó importante conocimiento y experiencias de campo en la implementación y evaluación de sistemas agroforestales en la Amazonía Peruana. El segundo autor agradece al fondo 2019 del Ministerio de Educación (MINEDU) como docente investigador de la Universidad Nacional Tecnológica de Lima Sur (UNTELS).

\section{REFERENCIAS BIBLIOGRÁFICAS}

Acuña, I. T. 2008. "Huella ecológica y biocapacidad: Indicadores biofísicos para la gestión ambiental. El caso de Manizales, Colombia." Revista Luna Azul 26:119-136.

Agbede, T. M., A. O. Adekiya, y J. S. Ogeh. 2014. "Response of soil properties and yam yield to Chromolaena odorata (Asteraceae) and 
Tithonia diversifolia (Asteraceae) mulches." Archives of Agronomy and Soil Science 60 (2):209-224.

Alegre, J. 2008. Manual de manejo de suelos con Agroforesteria. Lima (Perú): Universidad Nacional Agraria La Molina-

Alegre, J. 2017. “La Agroforestería en la Amazonía Peruana para recuperar suelos degradados y mitigar efectos de cambio climático.” En $X V I$ Congreso Nacional y VII Internacional de la Ciencia del Suelo. Ayacucho (Perú).

Alegre, J. C., M. R. Rao, L. A. Arevalo, W. Guzman, y M. D. Faminow. 2005. "Planted tree fallows for improving land productivity in the humid tropics of Peru." Agriculture, ecosystems y environment 110 (1-2):104-117.

Alegre, J., S. García, R. Vega, y Y. Arévalo. 2015. Manual reciclaje de nutrientes en sistemas agroforestales. Lima (Perú): Universidad Nacional Agraria La Molina-

Alegre, J., Y. Sánchez, D. Pizarro, y C. Gómez. 2019. Manejo de los suelos con sistemas silvopastoriles en las regiones de Amazonas y San Martín. Lima (Perú): Universidad Nacional Agraria La Molina.

Álvarez, L., y S. Ríos. 2009. Evaluación económica de plantaciones de tornillo; Cedrelinga catenaeformis, en el departamento de Loreto. Iquitos (Perú): Instituto de Investigaciones de la Amazonía Peruana (IIAP).

Alvim R., y P. K. R Nair. 1986. “Combination of cacao with other plantation crops: an agroforestry system in Southeast Bahia, Brazil." Agroforestry Systems4 (1): 3-15.

Arévalo L. A., J. C. Alegre, y R. Fasabi, R. 2003. "Efecto del fósforo sobre el establecimiento del centrosema macrocarpum benth dentro de una plantación de Pijuayo (bactris gasipaes hbk) en un ultisol del trópico húmedo." Ecología Aplicada 2 (1):93-97.

Arcila, J., F. Farfán, A. Moreno, L. F. Salazar, y E. Hincapié. 2007. Sistemas de producción de café en Colombia. Chinchiná (Colombia): Cenicafé.

Craven, D., D. Dent, D. Braden, M. S. Ashton, G. P. Berlyn, y J. S. Hall. 2011. "Seasonal variability of photosynthetic characteristics influences growth of eight tropical tree species at two sites with contrasting precipitation in Panama." Forest Ecology and Management 261 (10):1643-1653.

De Morais, V. T. M., L. A. G. Jacovine, C. M. M. E. Torres, E. B. B. M. Alves, H. N. de Paiva, R. Alcántara de la Cruz, y J. C. Zanuncio. 2019. "Early assessment of tree species with potential for carbon offset plantations in degraded area from the southeastern Brazil." Ecological indicators $98:$ 854-860.

Dilas, J. 2013. Identificación de oportunidades de Innovación tecnológicas en la producción del café en la región Cajamarca. Tesis de Maestría. Universidad Peruana Cayetano Heredia. Lima (Perú).

Dilas J. y N. Villanueva. 2014. Manual de producción de abono orgánico a partir de pulpa de café, utilizando microorganismos celulolíticos nativos. Lima (Perú): FIDECOM.

Farfán, F., J J. Arias, y N. M. Riaño. 2003. “Desarrollo de una metodología para medir sombrío en sistemas agroforestales con café." Cenicafe 54 (1): 24-34.

Galindo, V., Z. Calle, J. Chará, y I. Armbrecht. 2017. "Facilitation by pioneer shrubs for the ecological restoration of riparian forests in the Central Andes of Colombia." Restoration Ecology 25 (5): 731-737.

Gama, M. M. B., R. B. Rocha, A. K. D. Salman, A. M. Mendes, y M. R. Figueiró. 2013. "Reforestation feasibility in area formerly used for cattle rasing in the State of Rondônia, Northwest Brazilian Amazon." Revista Arvore 37(6): 1001-1010.

García, G., C. Paleo, J. Hernández, J. J. García, H. J. Muñoz, y C. G. Xavier. 2013. "Estimación de 
coeficientes y cocientes de forma para Pinus montezumae Lamb. En II simposio internacional en producción agroalimentaria tropical y XXV reunión cientifica-tecnológica forestal y agropecuaria tabasco. Tabasco (México).

Hohnwald S., J. Trautwein, A. P. Camarão, y C. B. Wollny. 2016. "Relative palatability and growth performance of capoeira species as supplementary forages in the NE-Amazon." Agriculture Ecosystems \& Environment 218: 107-115.

Hussain, A., H. Ramirez, W. M. Roca, y W. Bushuk. 1988. "Identification of cultivars of pasture legumes (Centrosema macrocarpum, $C$. pubescens and C. sp. n.) by acid polyacrylamide gel electrophoresis of cotyledons storage proteins." Euphytica 39 (2): $105-$ 107.

Jarrett, C., I. Cummins, y E. Logan. 2017. “Adapting indigenous agroforestry systems for integrative landscape management and sustainable supply chain development in Napo, Ecuador." En Integrating landscapes: Agroforestry for biodiversity conservation and food sovereignty. Montagnini, F. (ed). Cham (Suiza): Springer.

Julca, A., R. Carhuallanqui, N. J. Vera, S. B. Amez, R. C. Costa, C. Echevarría, y R. Borjas. 2010. Efecto de la sombra y la fertilización sobre las principales plagas del café var. "Catimor" en Villa Rica Pasco (Perú). Lima (Perú): Universidad Nacional Agraria La Molina.

Julca, A., Y. Ladera, L. Alvarado, R. Borjas, J. Vásquez, C. García, J. Jiménez, y V. Castro. 2018. Comportamiento de variedades brasileras en el banco de germoplasma de café en San Ramón-Chanchamayo. Lima (Perú): Universidad Nacional Agraria La Molina.

JNC (Junta Nacional de Café). 2019. Exportaciones y producción del café 2019, con malos resulta-

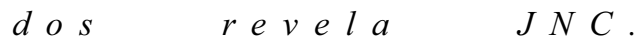

https://juntadelcafe.org.pe/exportaciones-yproduccion-de-cafe-2019-con-malosresultados-revela-jnc/ (Consultada el 18 de marzo de 2020).

Kairis O., C. Kosmas, C. Karavitis, C. Ritsema, L. Salvati, S. Acikalin, y A. Belgacem, 2014. "Evaluation and selection of indicators for land degradation and desertification monitoring: types of degradation, causes, and implications for management." Environmental management 54 (5): 971-982.

Layza R., F. Gonzales, y J. Terán. 2018. “La deforestación y el cambio climático en la provincia de San Martín periodo: 1973 al 2014.” Revista Cienciay Tecnologia 14(2): 19-30.

Lotutala, E. Y. y A. Kimbembi. 2018. "Mise en œuvre d'un système de production durable de manioc (Manihot esculenta Crantz) dans le village de Tadila en République Démocratique du Congo." Tropicultura 36 (4): 641-648.

Marquardt, K., R. Milestad, y L. Salomonsson. 2013. "Improved fallows: a case study of an adaptive response in Amazonian swidden farming systema." Agriculture and Human Values 30 (3): 417-428.

MINAGRI (Ministerio de Agricultura y Riego). 2017. El café peruano 2017. http://minagri.gob. pe/portal/485-feria-scaa/10775-el-cafeperuano (Consultado el 4 de febrero de 2019).

MINAM (Ministerio del Ambiente). 2015. Mapa nacional de cobertura vegetal: memoria $d e s c r i p t i v a$.

2015 . http://www.minam.gob.pe/patrimonionatural/wp-content/uploads/sites /6/2013/10/MAPA-NACIONAL-DEC O B E R T U R A - V E G E T A L FINAL.compressed.pdf (Consultada el 16 de marzo de 2020).

Milla, M., S. Oliva, S. Leiva, R. Silva, O. Gamarra, M. Barrena, y J. Maicelo. 2019. "Características morfológicas de variedades de café cultivadas en condiciones de sombra." Acta Agronómica 
$68(4): 271-277$

Moriones, M. L., y C. Montes. 2017. “Aporte de Tithonia diversifolia en abonos orgánicos: Efecto en producción y suelo en Cauca, Colombia." Biotecnología en el Sector Agropecuario y Agroindustrial 15 (2): 101-111.

Neina, D., A. Buerkert, y R. G. Joergensen. 2016. "Potential mineralizable $\mathrm{N}$ and $\mathrm{P}$ mineralization of local organic materials in tantalite mine soils." Applied Soil Ecology 108: 211-220.

Núñez, A. D. 2018. Estimación de biomasa aérea de Cedrelinga cateniformis Ducke en el Alto Mayo, San Martín. Tesis de grado. Universidad Nacional Agraria La Molina. Lima (Perú).

Olivares, G. R., y G. D. Torrejón. 2007. “Evaluación del potencial agroforestal de Colubrina glandulosa Perk (" shaina") en las provincias de Lamas y San Martin, Amazonia peruana." Revista Forestal del Perú 29 (1-2): 99-118.

Rondon, X. J., D. L. Gorchov, y R. B. Noble. 2009. "Projection of tree growth and timber volume following strip clear-cutting in the Peruvian Amazon." Forest ecology and management 257 (2): 588-599.

Theobald, T. F., F. Mussgnug, y M. Becker. 2014. "Live fences-a hidden resource of soil fertility in West Kenya." Journal of Plant Nutrition and Soil Science 177 (5): 758-765.

FAO (Organización de las Naciones Unidas para la Alimentación y la Agricultura). 2020. Portal $d e \quad s u e l o s \quad d e \quad l a \quad F A O$. http://www.fao.org/soils-portal/soildegradation-restoration/es/ (Consultada el 18 de marzo de 2020)

Pinto, T. T., G. E. Geisler, M. Santos, y M. T. S. Paulilo. 2020. "Germination of physically dormant seeds of Colubrina glandulosa Perkins (Rhamnaceae)." Brazilian Journal of Botany 43:91-97

Pretty J. 2007. “Agricultural sustainability: concepts, principles and evidence." Philosophical
Transactions of the Royal Society B: Biological Sciences 363 (1491): 447-465.

Reyes E., A. Bellagamba, J. J. Molina, L. Izquierdo, C. Deblitz, J. Chará, y E. Murgueitio. 2017. Measuring sustainability on cattle ranches: Silvopastoral systems. Bogotá (Colombia): CIPAV

Sánchez, P. A. 1987. "Soil productivity and sustainability in agroforestry system". En Agroforestry: A decade of development. Steppler, H. A. y P. K. Nair (eds). Nairobi (Kenia): ICRAF

Sánchez P., I. Ocampo, E. Parra, J. Sánchez, A María, A. Argumedo. 2017. "Evaluación de la sustentabilidad del agroecosistema maíz en la región de Huamantla, Tlaxcala, México." Agroecología 9: 111-122.

Sarandón, S. J. 2002. "El desarrollo y uso de indicadores para evaluar la sustentabilidad de los agroecosistemas". En Agroecología. El camino hacia una agricultura sustentable. Sarandón S. J. (ed). La Plata (Argentina): Ediciones Científicas Americanas

Sarandón, S. J., M. S. Zuluaga, R. Cieza, L. Janjetic, y E. Negrete. 2006. "Evaluación de la sustentabilidad de sistemas agrícolas de fincas en Misiones, Argentina, mediante el uso de indicadores." Agroecología 1: 19-28.

Silva, K. A., S. V. Martins, A. Miranda Neto, y W. H. Campos. 2015. "Semeadura direta com transposição de serapilheira como metodologia de restauração ecológica” Revista Árvore 39 (5): 811-820.

Somarriba, E., J. Beer, J. A. Orihuela, H. J. Andrade, R. Cerda, F. DeClerck, y L. Krishnamurthy. 2012. "Mainstreaming agroforestry in Latin America." En Agroforestry: the future of global land use. Nair, P. K. R. y D. Garrity (eds). Dordrech (Holanda): Springer.

SERNANP (Servicio Nacional de Áreas Naturales Protegidas por el Estado). 2017. Bosque de Protección Alto Mayo (BPAM). 
https://www.sernanp.gob.pe/alto-mayo (Consultada el 17 de marzo de 2020).

Suarez-Vasquez, S. 1994. "Pastures and soil recovery in the coffee producing areas of Colombia." En Seminario Manejo Integral de Suelos para una Agricultura Sostenida. Palmira (Colombia).

Vilas-Boas, H. F., L. F. Almeida, R. S. Teixeira, I. F. Souza, y I. R. Silva. 2018. "Soil organic carbon recovery and coffee bean yield following bauxite mining." Land Degradation \& Development 29 (6): 1565-1573.

Walentowski H., S. Heinrichs, S. Hohnwald, A. Wiegand, H. Heinen, M. Thren, O. A. Gamarra-Torres, A. B. Sabogal, y S. Zerbe. 2018. "Vegetation succession on degraded sites in the Pomacochas Basin (Amazonas, $\mathrm{N}$ Peru) - Ecological options for forest restoration." Sustainability 10 (3): 609.

Weber O. B., M. C. B. da Silva, C. F. da Silva, J. A. de Sousa, C. A. K. Taniguch, D. dos Santos Garruti, y R. E. Romero. 2019. "Biological and chemical attributes of soils under forest species in Northeast Brazil." Journal of Forestry Research 31: 1959-1973. 